select few well-funded and wellresourced regions.

*Rehan Ahmed Siddiquee Specialist Registrar in General Adult Psychiatry, West Midlands, email: rehon_007@hotmail.com, Arif A. Qadir Senior House Officer in Psychiatry, Cefn Coed Hospital, Swansea

doi: $10.1192 / p b .31 \cdot 10.398 b$

\section{Awareness of psychosexual problems in psychiatric patients among trainees in Pakistan}

Dr Raffi (Psychiatric Bulletin, June 2007,

31, 233-234) has raised some

interesting points about sexual dysfunction among patients of south-east Asian background. This is an issue which psychiatrists working in south-east Asian countries face routinely in their clinical practice.

We recently carried out a survey among psychiatric trainees in a teaching hospital in Peshawar, Pakistan enquiring about their awareness and understanding of psychosexual problems in psychiatric patients. Sixteen doctors (15 males, 1 female; age range 28-42 years) completed the questionnaire and although all were aware of the existence and likely causes of sexual dysfunction in psychiatric patients, only 6 said that they were routinely carrying out assessments of psychosexual functioning. Although most doctors (11 out of 16) felt comfortable about assessing such patients, they thought that patients were very uncomfortable talking about problems of such a sensitive nature. Nearly all doctors (14 out of 16) thought that the patients were unable to openly express themselves about their sexual problems. The most common problems identified by doctors were reduced libido, erectile dysfunction and premature ejaculation. Only 6 doctors thought that patients were getting adequate help for their psychosexual symptoms; the remaining 10 said that patients were just given reassurance.

All 16 doctors felt that there was a need for specific training for the adequate assessment and management of sexual dysfunctions in a manner which was culturally sensitive and acceptable to these patients. The stigma associated with psychiatric problems in general, owing to cultural and religious barriers, was further compounding the whole issue.

It is therefore pertinent that psychiatrists, especially in low- and middleincome countries, spend more time with patients to try to allay their anxieties about their psychosexual problems.

Rameez Zafar Consultant Psychiatrist Lincolnshire Partnership NHS Trust, email: rameez.zafar@|pt.nhs.uk, Khurram Sadiq Senior House Officer in Forensic Psychiatry, Lincolnshire Partnership NHS Trus

doi: $10.1192 / \mathrm{pb} .31 .10 .399$

\section{Crisis resolution \\ and home treatment teams and admissions}

Jethwa et al (Psychiatric Bulletin, May 2007, 31, 170-172) discussed several reasons for the $37.5 \%$ reduction in monthly admission following the formation of the crisis resolution and home treatment service in Leeds. The question remains: which one of these factors has the greatest influence? There is little doubt that screening the patient first by competent crisis resolution staff and the availability of home treatment helps to avoid inappropriate admissions. However, the formation of the service was at the same time as the $35 \%$ reduction of 54 general adult beds from 155 to 101. It is well known that if there are fewer beds the threshold for admission goes up and only the more severely ill and those on sections are admitted.

Unfortunately Jethwa et al did not provide other data which might help to explain the lower admission rates. These include occupancy rates before and after formation of the service, if rates of patients admitted under the Mental Health Act 1983 had increased, if consultants had difficulty in finding a bed or had to put patients on a waiting list for admission, and if alternative in-patient facilities were used (e.g. other respite beds, hostels, private hospitals, etc.). Perhaps the more likely explanation for the $37.5 \%$ reduction in admission rates is that there were 35\% fewer beds for admission and only the most severely ill were admitted.

Seng-Eng Goh Consultant in Old Age Psychiatry Bushey Fields Hospital, Dudley DY1 2LZ, email: Dr Seng-Eng.Goh@dudley.nhs.uk

doi: 10.1192/pb.31.10.399a

Authors' reply: We agree with Dr Goh that the $37.5 \%$ reduction in monthly admissions cannot be directly attributed to the implementation of the crisis resolution and home-based treatment service. Only high-quality randomised controlled trials can make unbiased assertions about the effectiveness of interventions without raising doubts about confounding variables. Such trials have already been conducted using operational definitions of 'crisis' and have demonstrated favourable results (Johnson et al, 2005). Unfortunately these studies often lack external validity. We believe that the strength of our study relates to its naturalistic design, as all patients were included. It is essentially a service evaluation which demonstrates the effectiveness of crisis resolution services in everyday clinical practice.

Dr Goh highlights potential confounding variables and we agree that many of these factors warrant further investigation. We are aware of no significant changes in the factors identified, in particular, the use of independent hospitals is often carefully regulated and their use is minimal given the financial implications. Unfortunately very few services introduce $24 \mathrm{~h}$ crisis resolution services without simultaneously closing in-patient beds. Our study must be considered in the context of recent randomised trials. When taking these into account we believe that crisis resolution and home-based treatment services reduce admission rates, although we accept that other variables may have an effect, and this requires further research.

JOHNSON, S., NOLAN, F., PILLING, S., et al (2005) Randomised controlled trial of acute mental health care by a crisis resolution team: the north Islington crisis study. BMJ, 331, 599-602.

*Krishma Jethwa Specialist Registrar in General Adult Psychiatry, South Hams CMHT, 8 Fore Street, Ivybridge, Devon PL21 9AB, email: dr.k.jethwa@ doctors.org.uk, Nuwan Galappathie Specialist Registrar in Forensic Psychiatry, Langdon Hospital Dawlish, Devon, Paul Hewson Senior Lecturer in Statistics, School of Mathematics and Statistics, University of Plymouth, Devon

doi: 10.1192/pb.31.10.399b 